\title{
Let's fight cancer: let-7 is a tool to enhance antitumor immune responses
}

\author{
Leonid A Pobezinsky*,1 \& Alexandria C Wells ${ }^{1}$ \\ ${ }^{1}$ Department of Veterinary \& Animal Sciences, University of Massachusetts, Amherst, MA 01003, USA \\ *Author for correspondence: Tel.: +1 413545 2393; Fax: +1 413545 632; Ipobezinsky@umass.edu
'Thus, manipulating let-7 levels in CTLs may be used as a new approach in T-cell-based therapies, defining let-7 miRNAs as a novel therapeutic target."

First draft submitted: 15 January 2018; Accepted for publication: 2 February 2018; Published online: 23 April 2018

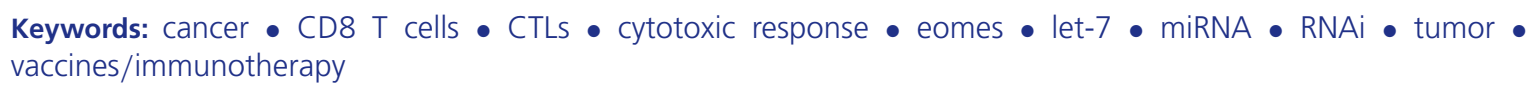

The ability to program cells into a particular state via the exploitation of global regulators of gene expression has significant therapeutic potential for many diseases, and thus has been a focus of biological research for several years. RNAi mediated by miRNAs is a strong candidate for such applications. MiRNAs are approximately 22 nucleotide (nt) noncoding RNAs that regulate gene expression by targeting specific mRNAs, in a sequence-specific manner, to prevent their translation into protein products. Since their initial discovery, over 2000 miRNA genes have been identified and demonstrated to regulate important cellular processes, including differentiation, proliferation and carcinogenesis. In this review, we will focus on one particular family of miRNAs, let-7, highlighting the role it plays in the differentiation of cytotoxic CD8 T lymphocytes (CTLs), and underscoring its potential for use in enhancing current immunotherapy strategies.

During the immune response, antigen-specific CD8 T lymphocytes undergo sequential changes that ultimately result in the acquisition of the cytotoxic function that is responsible for providing robust protection against viral infections and cancer. Before antigen encounter, mature CD8 T cells exist in a naive state, characterized by a quiescent phenotype in which cells use oxidative phosphorylation to generate energy, minimally proliferate, and have no cytotoxic function. Initial antigen recognition through the T-cell receptor, when accompanied by costimulation, leads to the activation of naive $\mathrm{T}$ cells. T-cell activation triggers a metabolic switch from oxidative phosphorylation to glycolysis, which supports lymphocytes as they rapidly proliferate and differentiate into CTLs. CTLs acquire the ability to kill target cells by secreting cytolytic effector molecules, such as perforin, granzymes and granulysin (in humans). Following clearance of the pathogenic cells, the majority of CTLs die by apoptosis, an event known as contraction. However, a small population of cells will survive, and will form antigen-specific memory CD8 T cells capable of providing long-term protection to the host, should antigen re-encounter occur [1]. Yet, in the case of chronic viral infection or cancer, these differentiation programs often become disrupted and CD8 $\mathrm{T}$ cells are rerouted into the so-called 'exhausted' state, defined by the inability of CD8 T cells to perform their cytotoxic function [2]. Identification of the signals responsible for disturbing the differentiation of CD8 T cells is currently a major focus of T-cell biology.

The tumor microenvironment (TME) provides signals that may suppress immune responses, including those mediated by CD8 T cells. The TME is a complex milieu of cells including not only tumor cells and CTLs but also such immunosuppressive cell populations as Tregs, myeloid-derived suppressor cells and subpopulations of tumor-associated macrophages, all of which are supported by the tumor-associated extracellular matrix [3]. In fact, these cellular components of the TME are capable of producing ligands, which lead to CD8 T-cell exhaustion upon recognition. Normally, activated/effector $\mathrm{T}$ cells express the receptors for these ligands where receptor/ligand engagement provides inhibitory signals to help prevent overactivation of $\mathrm{T}$ cells during the immune response, thus serving as a negative feedback. For this reason, these inhibitory receptors are often referred to as immune checkpoint receptors, of which there are many, the most well-known being PD-1, Tim-3 and CTLA-4 [2]. Inflammatory

Future Medicine 
conditions in the TME further drive the upregulation of the expression of these inhibitory receptors on the resident CTLs, in addition to promoting the expression of their ligands [3]. As a result, tumors provide robust inhibitory signals that drive CD8 T-cell exhaustion.

Immune checkpoint blockade therapy addresses this particular type of inhibitory signal by using antibodies to prevent signal transduction through specific inhibitory receptors. Blocking antibodies have been developed against many receptors and their ligands. For example, antibodies that target CTLA-4, the PD-1 receptor and its ligand PD-L1 have been approved by the US FDA and are in clinical use [4]. While this therapy has had immense success in the clinic, it has limitations. Primarily, the success of immune checkpoint blockade therapy is intrinsically tied to CD8 T-cell infiltration into the tumor. It is known that many so-called 'cold' tumors are weakly immunogenic and recruit very few immune cells to the TME [3]. Other immune-based therapies also have been developed to circumvent the constraints imposed by the TME. An important form of adoptive T-cell therapy centers on the isolation of tumor-specific CD8 T cells from the patient, expanding these tumor-infiltrating lymphocytes in vitro, and then transfusing the large number of tumor-specific CD8 T cells back into the patient, thus accounting for the failure to proliferate and expand. Moreover, this approach 'reverses' exhaustion as it removes cells from the suppressive TME and allows them to recover prior to reinfusion [3].

The use of these therapies in combination with each other has resulted in the significant improvement of patient responses; however, these improvements are observed in a limited number of patients, and the success rate is dependent on the type of cancer being treated. While the results are remarkable for patients that respond, in two recent clinical trials only $20 \%$ of melanoma patients showed durable clinical responses to ipilimumab (anti-CTLA-4 monoclonal antibody), and only $17 \%$ of non-small-cell lung cancer patients exhibited an objective response rate with nivolumab (anti-PD-1 monoclonal antibody) [5]. As such, it would be ideal to enhance the currently existing immunotherapies to increase the success rate for all patients, regardless of the type of cancer for which they are being treated. In fact, this is a specific goal of the Cancer Moonshot initiative established to address the prevention, early detection, treatment and curing of cancer [6].

\section{Let-7 miRNAs as global regulators}

MiRNAs regulate multiple important biological processes, including cell differentiation, through a posttranscriptional mechanism that is based on sequence-specific inhibition of mRNA translation. Genes-encoding miRNAs are first transcribed as a primary transcript by RNA PolII and PolIII, called primary miRNAs, which may vary in size, but are usually very long. While still in the nucleus, the primary miRNA is processed into the hairpinlike 60-70 nt precursor miRNA (pre-miRNA) by the microprocessor, a complex containing the endonuclease Drosha. Next, the pre-miRNA is exported from the nucleus to the cytoplasm, where the pre-miRNA stem loop is cleaved by the endonuclease Dicer, generating a mature approximately $22 \mathrm{nt}$ double-stranded miRNA. A single strand of this mature miRNA is then loaded into the RNA-induced silencing complex, which can prevent protein synthesis either by stalling the ribosomal machinery or by destabilizing the targeted mRNA [7]. The sequence with which miRNAs bind their RNA targets is known as the 'seed sequence', is typically 6-8 nt long, and is located at the $5^{\prime}$-end of the miRNA. Based on the conservation of this seed sequence, miRNAs are grouped into families, such that all members of one miRNA family have the same target mRNAs.

Let-7 is one of the most highly conserved families of miRNAs in the animal kingdom. Having undergone several duplications in evolution, the let-7 family is comprised of multiple paralog genes expressed on different chromosomes, forming the largest miRNA family in mammals. In fact, sequences of mature let-7 miRNAs are often identical although they are derived from different precursors and genes [8]. To indicate the differences in sequence across genes, a letter is placed after let-7 (i.e., let-7a, let-7b), and a number is placed after this letter to indicate that the same sequence of gene is expressed in multiple genomic locations (i.e., let-7c-1, let-7c-2). In humans, there are ten mature let- 7 family members generated from 13 precursor sequences, and in mice there are eight mature family members arising from 11 precursor miRNAs.

It has been demonstrated that let-7 miRNAs are involved in multiple biological processes including differentiation, cell death and metabolism [8,9]. Specifically, let-7 miRNAs were identified as potent tumor suppressors that directly target mRNAs of genes involved in the cell cycle and in signal transduction pathways that lead to carcinogenesis [8]. Although let-7 miRNAs can be found in many types of cells and tissues, the expression levels vary, revealing complicated regulation. In fact, let-7 miRNA expression is post-transcriptionally regulated by multiple factors that control different stages of let-7 biogenesis. Lin28 and Lin28B are well-studied fetal proteins, that block the generation of mature let-7 miRNAs. Both proteins interfere with Dicer processing by binding to a highly 
conserved sequence within the stem loop of let-7 miRNA precursors and recruiting the terminal uridylyl transferases, which uridylate the immature miRNA, allowing the exonuclease Dis3I2 to recognize and degrade it [10,11]. This particular mechanism is used to inhibit global let-7 expression during early embryogenesis. Lin28-mediated modulation of let-7 miRNA levels has been implicated during early embryogenesis and in establishing the immune responses of neonates $[8,12]$.

\section{Let-7 miRNAs in T-cell responses}

We and others have shown that the let-7 miRNAs are expressed in T-cell progenitors and that their expression is very abundant in mature $\mathrm{T}$ lymphocytes, suggesting a potentially important role for these miRNAs in T-cell-mediated immune responses $[13,14]$. In fact, the regulatory role of let-7 miRNAs has been demonstrated during the activation and differentiation of CD4 T-cell subsets. Specifically, it has been suggested that the let-7 miRNAs are in part responsible for inhibiting the expression of proteins in the mTOR pathway, the major metabolic hub in activated and effector CD4 T cells [15]. Recently, it has been reported that Tregs may even prevent the differentiation of CD4 T-helper cells by secreting let-7 containing exosomes [16]. Thus, the ability of let-7 to modulate CD4 T-cellmediated responses, including the differentiation of IFN- $\gamma$, IL-4 and IL-17-producing CD4 T-helper cells, has become increasingly clear $[15,17,18]$.

Although the expression of let-7 miRNAs in CD8 T lymphocytes has been shown earlier, the role of these miRNAs in regulation of CD8 T-cell differentiation was not clear [13]. In our recent paper, we demonstrated the significance of let-7 expression for the homeostasis and function of CD8 T lymphocytes. This work is based on a finding, which is in agreement with previous observations [13] that antigen stimulation of CD8 T cells results in a profound inhibition of let-7 expression. Two important questions followed this observation: why do naive CD8 $\mathrm{T}$ cells require high levels of let-7 miRNA expression and why must activated lymphocytes downregulate these miRNAs? These questions were addressed in experiments where let-7 levels in T cells were genetically manipulated. Let-7 depletion was achieved using previously described mice with a T-cell-specific Lin 28 transgene while let-7 reexpression was induced in $\mathrm{T}$ cells derived from doxycycline inducible let-7 transgenic (let-7Tg) mice $[9,19]$.

Analysis of naive CD8 T cells with lin28-mediated knockdown of let-7 expression revealed compromised homeostasis of these cells in vivo. Due to an unknown molecular mechanism, let-7-deficient naive T cells exhibited spontaneous activation and increased proliferation resulting in the loss of the quiescent state. These unexpected results led to the model that let-7 miRNAs function as a molecular brake that prevents spontaneous CD8 Tcell activation, while antigen stimulation releases it by actively reducing let-7 expression and allowing $T$ cells to differentiate into killer cells. In fact, the extent of functional maturation inversely correlates with the levels of let-7 expression in effector cells. CTLs with let-7-deficiency exhibited enhanced cytotoxic function in vitro, while let-7Tg CTLs failed to lyse target cells as compared with their wild-type counterparts. In vivo, let-7Tg mice were unable to mount an efficient antiviral response and failed to reject an allogeneic tumor, a response that typically results in the activation of $1-3 \%$ of all CD8 T cells and immediate rejection of the grafted cells. Thus, the reduction of let-7 expression results in enhanced $\mathrm{T}$ lymphocyte function while high levels prevent it.

We have only begun to understand the complexity of let-7-mediated regulation of CD8 T-cell differentiation, in which the let-7 miRNAs control multiple levels of this process, including the proliferation, metabolism and acquisition of effector functions. Specifically, the let-7 miRNAs inhibit expression of cell-cycle proteins, and the transcription factor Myc, which is responsible for inducing many of the genes that are involved in proliferation and glycolysis [20]. Furthermore, let-7-mediated targeting of the transcription factor Eomes suppresses the effector function of activated CD8 T cells. This work clearly demonstrated that the let-7 miRNAs are important regulators of CD8 T-cell differentiation and function.

Importantly, in the absence of the let-7 miRNAs, antigen-specific CTLs exhibited dramatically enhanced cytotoxic function. Thus, manipulating let-7 levels in CTLs may be used as a new approach in T-cell-based therapies, defining let-7 miRNAs as a novel therapeutic target. We expect to significantly improve T-cell performance in chronic viral and antitumor responses by decreasing the levels of let-7 miRNAs and enhancing the killer-function of T lymphocytes. Specifically, this approach could be very useful in improving the performance of existing cancer immunotherapies, which include immune checkpoint blockade, adoptive T-cell transfer and even CAR-T-cellbased therapies. Such manipulations of let-7 levels in vitro and in vivo are already feasible and can be accomplished through cell-targeted biodelivery of agents that can deplete mature let-7 miRNAs in tumor-specific $\mathrm{T}$ cells.

To successfully translate these findings into clinical practice and use them as a therapeutic tool that enhances antitumor immune responses, more fundamental and preclinical studies will be required. At the moment, it is not 
clear how the tumor immunosuppressive environment will affect the function and survival of let-7-deficient CTLs in vivo, despite the unexpected and gratifyingly potent performance of these cells in vitro. Furthermore, based on the high conservation of let-7 miRNAs and their targets, the similarity of let-7-mediated effects in mouse and human CD8 $\mathrm{T}$ cells can be predicted, but has yet to be shown. Whether the let-7 miRNAs are involved in the formation of memory $\mathrm{T}$ cells is unknown. However, the discovery of such an involvement would be of great interest and could have important implications for the development of more effective prophylactic and therapeutic cancer vaccines, thus contributing to cancer prevention and treatment, both of which are identified as goals of the aforementioned 'Cancer Moonshot' program [6]. The development of appropriate tumor models, including humanized mice, will be needed to address these important questions. Taken together, the development of let-7-based therapies could significantly advance the field of immunotherapy by eliminating or improving the limitations of the currently available immunotherapies, while at the same time maintaining the integrity of their most effective features. While let-7 has become a potential therapeutic target, ironically nothing is known about its regulation in T cells. Thus, understanding the molecular mechanisms that control let-7 miRNA expression during CD8 T-cell differentiation is yet another aspect of let-7 immunology that should command urgent attention.

In conclusion, the discovery of the let-7-mediated molecular machinery that regulates cytotoxic T-cell function provides information about new levels of control in the immune system and identifies additional targets for the manipulation of T-cell immune responses. While there is still much to learn about the role of let- 7 in T cells, and other hematopoietic cells, the potential of let- 7 as a therapeutic target is promising and extremely exciting.

Financial \& competing interests disclosure

The authors have no relevant affiliations or financial involvement with any organization or entity with a financial interest in or financial conflict with the subject matter or materials discussed in the manuscript. This includes employment, consultancies, honoraria, stock ownership or options, expert testimony, grants or patents received or pending, or royalties.

No writing assistance was utilized in the production of this manuscript.

Open access

This work is licensed under the Attribution-NonCommercial-NoDerivatives 4.0 Unported License. To view a copy of this license, visit http://creativecommons.org/licenses/by-nc-nd/4.0/

\section{References}

1. Kaech SM, Cui W. Transcriptional control of effector and memory CD8+ T cell differentiation. Nat. Rev. Immunol. 12(11), 749-761 (2012).

2. Blackburn SD, Shin H, Haining WN et al. Coregulation of CD8+ T cell exhaustion by multiple inhibitory receptors during chronic viral infection. Nat. Immunol. 10(1), 29-37 (2009).

3. Joyce JA, Fearon DT. T cell exclusion, immune privilege, and the tumor microenvironment. Science 348(6230), 74-80 (2015).

4. Sharma P, Allison JP. The future of immune checkpoint therapy. Science 348(6230), 56-61 (2015).

5. Topalian SL, Drake CG, Pardoll DM. Immune checkpoint blockade: a common denominator approach to cancer therapy. Cancer Cell 27(4), 450-461 (2015).

6. $\quad$ Singer DS, Jacks T, Jaffee E. A U.S. "Cancer Moonshot" to accelerate cancer research. Science 353(6304), 1105-1106 (2016).

7. Ha M, Kim VN. Regulation of microRNA biogenesis. Nat. Rev. Mol. Cell Biol. 15(8), 509-524 (2014).

8. Bussing I, Slack FJ, Grosshans H. let-7 microRNAs in development, stem cells and cancer. Trends Mol. Med. 14(9), 400-409 (2008).

9. Zhu H, Shyh-Chang N, Segrè AV et al. The Lin28/let-7 axis regulates glucose metabolism. Cell 147(1), 81-94 (2011).

10. Heo I, Joo C, Kim YK et al. TUT4 in concert with Lin28 suppresses microRNA biogenesis through pre-microRNA uridylation. Cell 138(4), 696-708 (2009).

11. Faehnle CR, Walleshauser J, Joshua-Tor L. Mechanism of Dis312 substrate recognition in the Lin28-let-7 pathway. Nature 514(7521), 252-256 (2014).

12. Wang J, Wissink EM, Watson NB et al. Fetal and adult progenitors give rise to unique populations of CD8+ T cells. Blood 128(26), 3073-3082 (2016).

13. Wu H, Neilson JR, Kumar P et al. miRNA profiling of naive, effector and memory CD8 T cells. PLoS ONE 2(10), e1020 (2007).

14. Wells AC, Daniels KA, Angelou CC et al. Modulation of let-7 miRNAs controls the differentiation of effector CD8 T cells. Elife 6, pii: e26398 (2017).

15. Marcais A, Blevins R, Graumann J et al. microRNA-mediated regulation of mTOR complex components facilitates discrimination between activation and anergy in CD4 T cells. J. Exp. Med. 211(11), 2281-2295 (2014). 
16. Okoye IS, Coomes SM, Pelly VS et al. MicroRNA-containing T-regulatory-cell-derived exosomes suppress pathogenic T helper 1 cells. Immunity 41(1), 89-103 (2014).

17. Polikepahad S, Knight JM, Naghavi AO et al. Proinflammatory role for let-7 microRNAS in experimental asthma. J. Biol. Chem. 285(39), 30139-30149 (2010).

18. Guan H, Fan D, Mrelashvili D et al. MicroRNA let-7e is associated with the pathogenesis of experimental autoimmune encephalomyelitis. Eur. J. Immunol. 43(1), 104-114 (2013).

19. Pobezinsky LA, Etzensperger R, Jeurling $S$ et al. Let-7 microRNAs target the lineage-specific transcription factor PLZF to regulate terminal NKT cell differentiation and effector function. Nat. Immunol. 16(5), 517-524 (2015).

20. Wang R, Dillon CP, Shi LZ et al. The transcription factor Myc controls metabolic reprogramming upon T lymphocyte activation. Immunity 35(6), 871-882 (2011). 
